\title{
Atmospheric dynamics in long-period Cepheids
}

\section{$\mathrm{H} \alpha$ profile variations}

\author{
D. Gillet

\begin{abstract}
Observatoire de Haute-Provence - CNRS/PYTHEAS/Université d'Aix-Marseille, 04870 Saint Michel l'Observatoire, France
\end{abstract} \\ e-mail: denis.gillet@oamp.fr
}

Received 22 January 2014 / Accepted 13 June 2014

\section{ABSTRACT}

\begin{abstract}
Context. Despite recent time series of very high quality of $\mathrm{H} \alpha$ profile variations of classical Cepheids, the dynamics of the pulsating atmosphere of long-period Cepheids is not yet understood.

Aims. Taking into account the propagation of intense shock waves in the atmosphere, we propose a general scheme of the atmospheric dynamics in long-period Cepheids.

Methods. We use a time series of $\mathrm{H} \alpha$ profiles of the long-period Cepheid X Cygni and HARPS high-resolution spectroscopy of three long-period Cepheids that have not yet been fully interpreted.

Results. Caused by the main shock occurring at each pulsation cycle, the $\mathrm{H} \alpha$ profile first presents a blueshifted component in emission or in absorption depending on the shock intensity. Then, when the shock is detached from the photosphere, the emission shifts on the red side of the line profile which now has the form of a P Cygni profile. A second shock is also observed. It is the consequence of the ballistic motion of the atmosphere. Thus, the interaction of the main shock with infalling layers can qualitatively explain observed variable peculiairities in the $\mathrm{H} \alpha$ profile.

Conclusions. The evolution of the $\mathrm{H} \alpha$ profile occurring in long-period Cepheids is similar to the evolution observed in radially pulsating stars such as RR Lyrae stars, in which the intensity of the main shock is strong enough to induce a propagation away from the photosphere.
\end{abstract}

Key words. stars: variables: Cepheids - stars: atmospheres - shock waves

\section{Introduction}

Today, the basic mechanism of the pulsation of classical Cepheids is well understood. However, the dynamics of the atmosphere of long-period Cepheids, which are high-luminosity stars, is increasingly complex. For instance, Nardetto et al. (2008), after having analyzed very high-quality time series of $\mathrm{H} \alpha$ profile variations of eight Cepheids, concluded that profiles of long-period Cepheids exhibit an enigmatic spectroscopic behaviour, which is extremely difficult to explain and requires further theoretical investigations.

As shown by Neilson \& Lester (2008), radial pulsations and strong shocks propagating in the atmosphere play a major role in the enhancement of mass loss in classical Cepheids. Calculations indicate that mass-loss rates range from $10^{-10} M_{\odot} \mathrm{yr}^{-1}$ to $10^{-7} M_{\odot} \mathrm{yr}^{-1}$, larger than if the mass-loss wind was driven by radiation alone. Thus, our understanding of the dynamics occurring in extended atmospheres of classical Cepheids, especially the physics of the shock propagation, could provide reasonable estimates of the shock-induced mass loss.

The presence of compact circumstellar envelopes has been detected by interferometry around several long-period Cepheids, if not all (e.g. Kervella et al. 2009). These warm circumstellar envelopes are certainly created by ongoing mass loss induced by pulsation and shocks, while cold dust shells, if they exist, are most likely of interstellar origin. Thus, our understanding of the shock dynamics occurring in these extended atmospheres is also a key to explaining the formation of warm circumstellar envelopes.

High-resolution $(R \equiv \lambda / \Delta \lambda \gtrsim 20000)$ spectroscopic observations of classical Cepheids have been done by many authors.
Krap (1960) was the first to report bizarre behavior of $\mathrm{H} \alpha$ in X Cygni during the whole pulsation cycle. This bizarre behaviour appears to be characteristic in Cepheids of periods greater than about ten days. From 19 spectra of $10 \AA / \mathrm{mm}$, Wallerstein (1983) observed in X Cygni a redward displaced component of $\mathrm{H} \alpha$ near the pulsation phase $\varphi=0.80$ and a violetshifted component near maximum which may be related to Ca II emission seen before maximum. Wallerstein concluded that the physical origin of these components, which are induced by strong velocity gradients occurring in the outer envelope of the star, is not readily apparent. This means that the intriguing $\mathrm{H} \alpha$ variations are not in accord with the classical pulsation model, in which the motion of all atmospheric layers is almost synchronized as they are in short-period and low-luminosity Cepheids like $\delta$ Cephei. Butler \& Bell (1997) showed that the amplitude of the $\mathrm{H} \alpha$ radial velocity curve is completely out of phase with the standard Fe I radial velocity curve of short-period Cepheids, in which only slightly differences are observed.

Kiss \& Vinkó (2000), using high-resolution spectroscopic observations of 18 bright Cepheids $(R=40000)$, find that the smallest full-width at half maximum (FWHM) always occurs very close to the phase of maximal radius, while the largest FWHM is observed just before the smallest radius i.e. when the global compression of the atmosphere is the strongest near phase $\varphi=0.80-0.85$. These results confirm the previous determinations of the variation of the turbulent velocity throughout the pulsation cycle (see Breitfellner \& Gillet 1993a, and references therein). However, all these results apply to the case of fundamental pulsators. For overtone Cepheids, the largest FWHM is shifted later $(\varphi=0.95-1.00)$, just before the luminosity maximum. 
Using 288 high-resolution $(R=49000)$ spectra of the classical Cepheid star $\delta$ Cephei, and non-linear, non-adiabatic pulsational models, Gillet et al. (1999) determined the microturbulence velocity curve with a high temporal resolution. The turbulent velocity reaches its maximum, about $7 \mathrm{~km} \mathrm{~s}^{-1}$, at minimum radius and decreases to $2 \mathrm{~km} \mathrm{~s}^{-1}$ during the expansion of the star, as expected. Moreover, in addition to the global compression effect, the different shock waves propagating in the atmosphere during a pulsation period induce at each time a turbulence amplification, which increases with the shock amplitude. These results clearly show that the propagation of progressive waves and shocks plays a basic role in the dynamics of the atmosphere especially in a very extended atmosphere like that of long-period Cepheids.

Unlike short-period Cepheids, two large and consecutive accelerations occur in the atmosphere of X Cygni at each pulsation cycle. Breitfellner \& Gillet (1993b) showed that these accelerations correspond to two strong peaks of turbulence. The smallest peak is induced by the global atmospheric compression. This is the one we see in short-period Cepheids. The strongest peak occurs early near phase $\varphi=0.75-83$, depending on the absorption line used to calculate the turbulent velocity. Breitfellner \& Gillet (1993b) suggested that the physical origin of this enigmatic peak must be the consequence of strong density variations occurring in the stellar atmosphere during the pulsation, but they do not specify more precisely the mechanism responsible.

In Sect. 2 of this paper, we briefly describe the observations of the $\mathrm{H} \alpha$ line profile of X Cygni. Sections 3-7 present a general interpretation of the complex $\mathrm{H} \alpha$ profile observed for both $\mathrm{X}$ Cygni $(P=16.4 \mathrm{~d})$ and $\ell$ Carinae $(P=35.6 \mathrm{~d})$. Section 8 is devoted to the physical origin of the two atmospheric accelerations occurring in each pulsation cycle. The variations of the turbulent velocity is discussed in Sect. 9. A comparison with other radially pulsating stars is given in Sect. 10. Finally, some concluding remarks are given in Sect. 11.

\section{Observations and data processing}

Twenty-three spectra of the long-period Cepheid X Cygni, centred on $6580 \AA$, including $\mathrm{H} \alpha$ were observed at the $1.52 \mathrm{~m}$ telescope of the Observatoire de Haute-Provence between July 1989 and September 1992. These spectra were acquired during the thesis work of M.G. Breitfellner (Breitfellner 1993). The spectrograph AURELIE at a resolving power $R=60000$ was used. A detailed description of the observations and the data reduction is given by Breitfellner \& Gillet (1993a) and the journal of the spectroscopic observations of X Cygni is given in Table 2 of Breitfellner \& Gillet (1993b) with the exception of the spectra at pulsation phases $\varphi=0.396$ and 0.945 which were realised a little later. The $\mathrm{H} \alpha$ profiles are given in Fig. 1. Wavelengths are in the stellar restframe, using a systemic radial velocity of X Cygni $V_{*}=9.8 \mathrm{~km} \mathrm{~s}^{-1}$ which was given by the General Catalogue of Stellar Radial Velocities (Wilson 1953). From a radial-velocity curve based on 17 McDonald Observatory coudé spectra, Abt (1978) obtained $V_{*}=9.7 \mathrm{~km} \mathrm{~s}^{-1}$. Later, Caldwell \& Coulson (1987) obtained $V_{*}=7.9 \mathrm{~km} \mathrm{~s}^{-1}$ and Moffett \& Barnes (1987) $V_{*}=10.5 \mathrm{~km} \mathrm{~s}^{-1}$ while Breitfellner \& Gillet (1993b), from the observation of four iron lines at high spectral resolution $(R=60000)$ which are neither blended by other lines nor perturbed by emission component, found an average value of $V_{*}=8.0 \pm 0.6 \mathrm{~km} \mathrm{~s}^{-1}$. Finally, the systemic radial velocity of X Cygni seems close to the value that Breitfellner \& Gillet (1993b) obtained and, accordingly, the position of the laboratory wavelength in Fig. 1 is correctly marked.

\section{Overview of $\mathrm{H} \alpha$ profile variations}

The $\mathrm{H} \alpha$ line is one of the strongest whose core is formed in the highest atmospheric layers. Figure 1 shows observed profiles of the $\mathrm{H} \alpha$ line for X Cygni. At first glance, the variation of the $\mathrm{H} \alpha$ profile is complex throughout the pulsation cycle and reveals very strange behaviour that is not consistent with usual short-period Cepheid pulsation. Several spectral phenomena are striking. The first is the presence of a small emission between the pulsation phases 0.062 and 0.580 i.e. from maximum brightness to minimum brightness. This emission component is redshifted and its shift decreases when phase increases.

The second surprising feature is the presence of a blueshifted absorption component from approximately the phase 0.880 to the maximum extension of the atmosphere occurring near phase 0.40 . It reaches its maximum depth at the phase 0.2 . The width of this absorption component is up to twice that of the redshifted component. The two absorption components appear as a double line until the phase 0.30 . Near phase 0.40, the deeper and permanent absorption component, which was first redshifted, returns to the laboratory wavelength.

The third and last striking spectral feature is the presence of a double absorption line between phases 0.702 and 0.942 . This line doubling is probably not a reverse Schwarzschild phenomenon, because, unlike the classical picture of the Schwarzschild mechanism (Schwarzschild 1952), the laboratory wavelength is not located between the two absorption components, but it is centred on the component with the shortest wavelength.

Around the phase 0.642 , the line profile is almost normal i.e. virtually symmetrical, without double-absorption or emission components. In addition, the profile is centred at the laboratory wavelength. This relaxed profile occurs at the luminosity minimum when the photosphere is at half its infalling movement.

The H $\alpha$ profile of $\delta$ Cephei, like other short-period Cepheids, does not show these three particular features. There are no emission lines or double-absorption lines (Breitfellner \& Gillet 1993a). Changes in line profile patterns of short-period Cepheids are consistent with the classical scheme of stellar pulsation in which the motion of atmospheric layers is close to a standing wave. On the contrary, the atmospheric dynamics occurring in long-period Cepheids seems to be quite complex. The pulsation certainly has the form of a progressive wave, sometimes with a de-synchronization of the motion of atmospheric layers and the propagation of strong shock waves, which must induce a large atmospheric extension.

\section{The $\mathrm{H} \alpha$ blueshifted absorption/emission component}

For X Cygni, a blueshifted absorption component appears within the $\mathrm{H} \alpha$ profile from phase 0.880 (Fig. 1), but, unlike $\ell$ Carinae, no emission component above the continuum is observed until the disappearance of this absorption around phase 0.4 . For $\ell$ Carinae, instead of this absorption, a blueshifted emission is observed above the continuum approximately from phase 0.75 to 0.85 (Baldry et al. 1997; Nardetto et al. 2008). Its blueshift is close to $55 \mathrm{~km} \mathrm{~s}^{-1}$.

Fokin et al. (2009) calculated a nonlinear radiative pulsating model for the long-period Cepheid $\ell$ Carinae. During the beginning of their propagation in the lower atmosphere, several shocks merge into a single shock, hereafter called the main shock (for a detailled discussion about shock formation processes see Fokin et al. 1996). At this time, the shock amplitude is already high 
D. Gillet: Atmospheric dynamics in long-period Cepheids

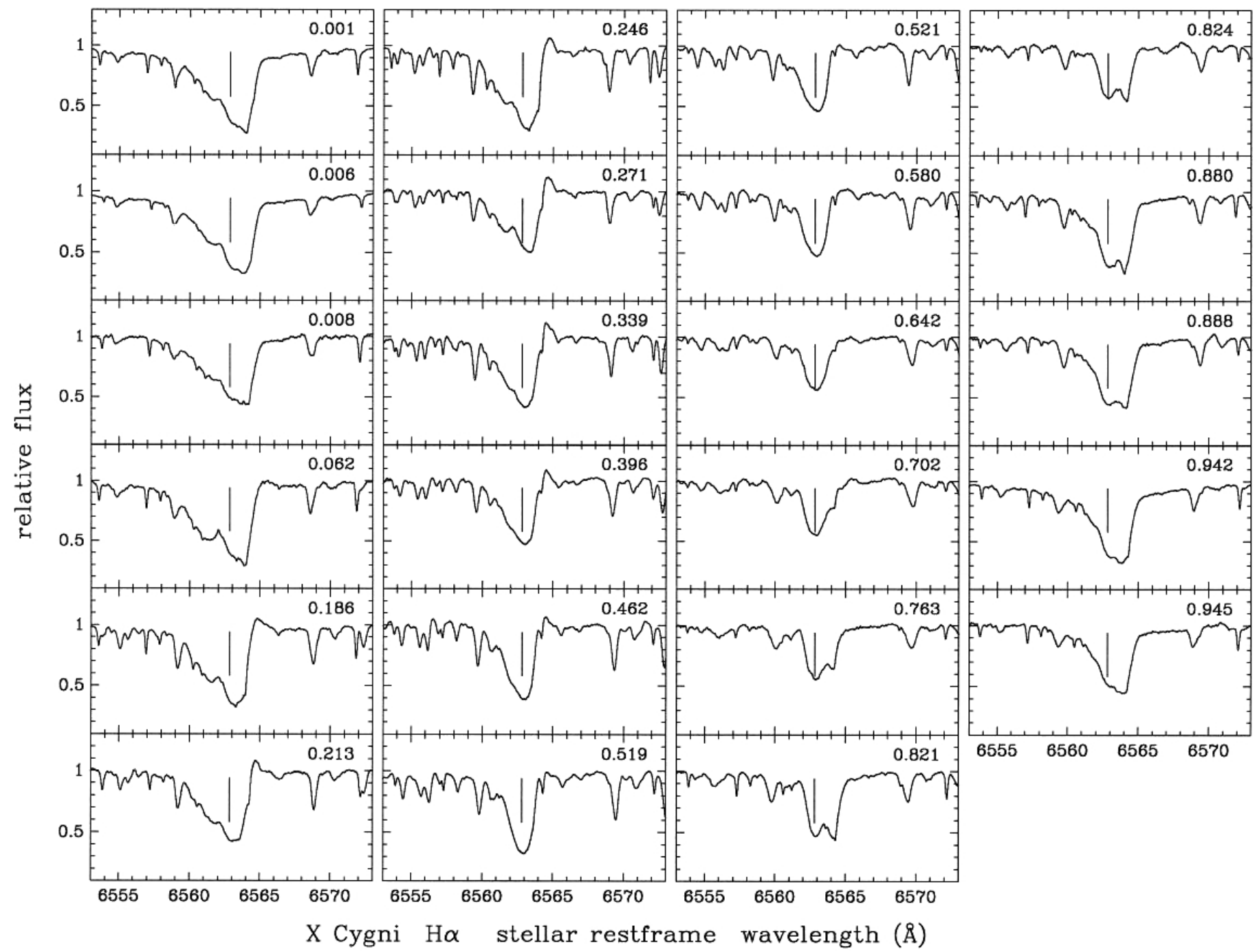

Fig. 1. Observed profiles of the H $\alpha$ line for X Cygni between July 1989 and September 1992 at the $1.52 \mathrm{~m}$ telescope of the Observatoire de HauteProvence. The pulsation phase $\varphi$ is denoted in the upper corner of each spectrum where the wavelength is given in $\AA$ in the stellar rest frame and the small vertical lines give the position of the laboratory wavelength of $\mathrm{H} \alpha$. The spectrograph AURELIE at a resolving power $R=60000$ was used. This figure was produced by M.G. Breitfellner during his thesis work (Breitfellner 1993).

when the mass-per-area depth of the shock $\Delta m_{\text {shock }} / 4 \pi R_{\text {shock }}^{2}$ has values between $10 \mathrm{~g} \mathrm{~cm}^{-2}$ and $100 \mathrm{~g} \mathrm{~cm}^{-2}$. This is not the case for RR Lyrae stars, because the intensity of the main shock is high only when its mass-per-area depth becomes very small i.e. when the shock reaches the highest part of the atmosphere. Consequently, in long-period Cepheids, the shock Mach number is moderate and cannot reach the large values $(\sim 30)$ observed in RR Lyrae stars (see Fig. 3 of Fokin \& Gillet 1994). This probably explains why no emission component is observed in X Cygni $\left(\sim 9 M_{\odot}\right)$ and why only a small blueshifted emission is detected for $\ell$ Carinae $\left(\sim 15 M_{\odot}\right)$.

It is worth noting that the shock amplitude $\Delta u=u_{2}-u_{1}$, where $u_{1}$ and $u_{2}$ are the LTE pre- and postshock velocities respectively, is a rough approximation. Because of the use of the artificial viscosity, $u_{2}$ corresponds to the velocity in the postshock region where recombinations occurs. This means that we do not have access to the postshock velocity just after the viscous shock front and consequently to the shock front velocity $v_{\mathrm{s}}$ itself. According to Fadeyev \& Gillet (2004), the gas emitting the Balmer line radiation is located at the rear of the shock wave in the hydrogen recombination zone, where the gas flow velocity in the frame of the observer is approximately one-half of the shock wave velocity: $u_{2} \simeq v_{\mathrm{s}} / 2$. Thus, the shock amplitude is probably underestimated until a factor of two.

\section{The $\mathrm{H} \alpha$ redshifted emission: the P Cygni profile}

The shock wave called the main shock, induced by $\kappa$-mechanisms of $\mathrm{H}, \mathrm{He} \mathrm{I}$, and He II, is the strongest shock occurring in the atmosphere during a pulsation cycle. If we assume that it is already located above the photosphere just after the luminosity maximum (pulsation phase $\varphi=0.062$ ), and if it is sufficiently detached from it, then as already discussed by Gillet \& Fokin (2014), the $\mathrm{H} \alpha$ line appears as a P Cygni profile. This is the profile that we observe (Fig. 1). After the phase 0.5, the P Cygni profile becomes almost perfect in the sense that the absorption is composed of a single component centered at the restframe wavelength. Thus, following Gillet \& Fokin (2014), the shock front velocity would be $70 \mathrm{~km} \mathrm{~s}^{-1}$ during the terminal phase of the radiative shock propagation. Just after the luminosity maximum, the $\mathrm{H} \alpha$ profile is a double absorption with a redshifted component associated with the $\mathrm{P}$ Cygni profile. Then, from the luminosity maximum until phase 0.580 , the velocity of the latter absorption component gradually returns to 


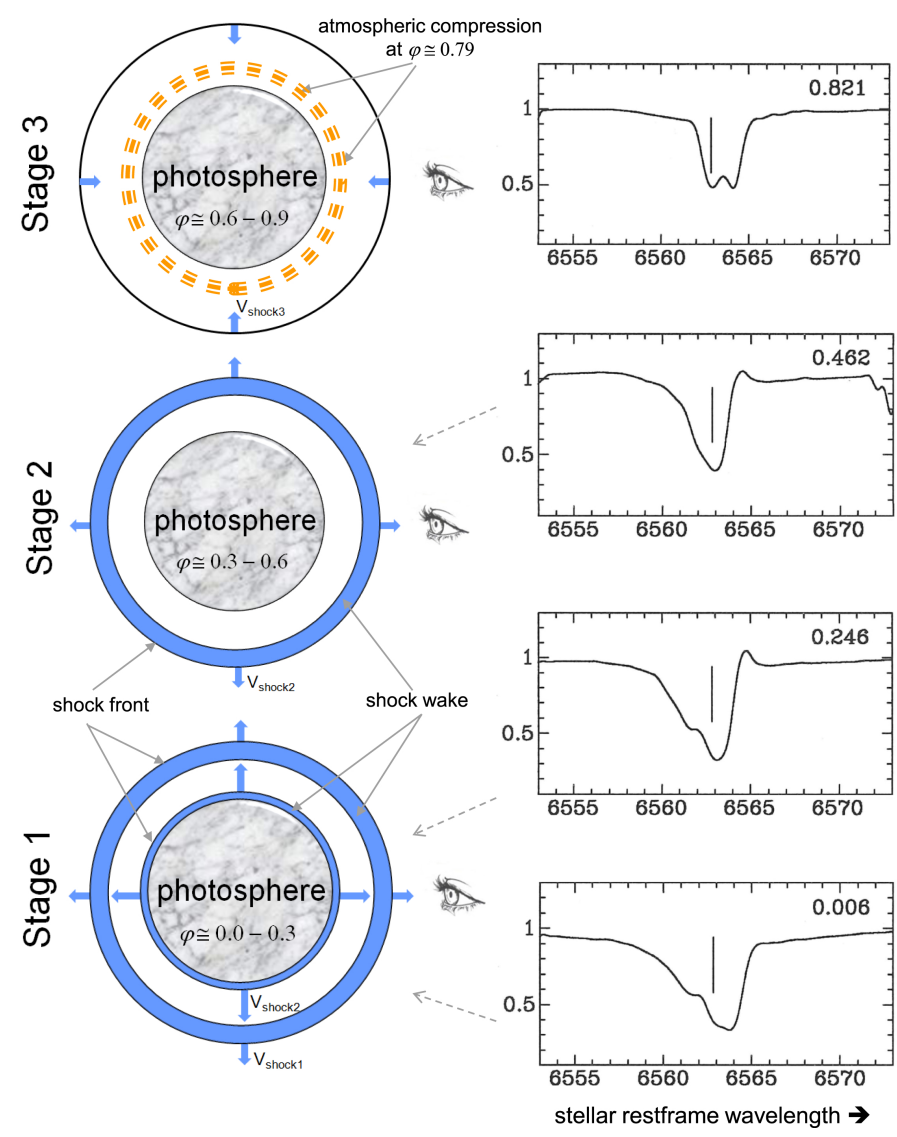

Fig. 2. Hypothetical scheme of evolution of the $\mathrm{H} \alpha$ line profile when the main shock wave propagates in the envelope of the long-period Cepheid $\mathrm{X}$ Cygni. Stage 1 begins around the luminosity maximum i.e. when the main shock front induced by the current pulsation cycle (shock 2) is emerging from the photosphere. In addition, from phase 0.062 , the main shock front of the previous cycle becomes visible because of the appearance of a P Cygni profile, which means that the shock front (shock 1) is well detached from the photosphere. At that time, two shock waves are simultaneously visible. During the second stage (pulsation phases between 0.3 and 0.6 ), only the main shock of the current pulsation cycle is visible (shock 2). In this interval of phases, it is well detached from the photosphere and consequently, the $\mathrm{H} \alpha$ line is now a P Cygni profile. Finally, the third and last stage (pulsation phases between 0.6 and 0.9 ) shows the infalling shock (shock 3 ) induced by atmospheric layers falling by gravity on the photosphere. The expanding shell (blue or gray area), just behind the shock front, is actually the radiative wake of the shock in which is formed the $\mathrm{H} \alpha$ emission line. Line profiles are clean profiles derived from observations of Fig. 1.

the zero velocity. Just after the disappearance of the emission (phase 0.642), the $\mathrm{H} \alpha$ profile becomes a nice single Gaussian, almost perfectly centered on the zero velocity. If we assume that the blended redshifted absorption by the blueshifted absorption, just after the luminosity maximum, is actually centered on zero velocity, then the shock front velocity decreases from $100 \mathrm{~km} \mathrm{~s}^{-1}$ to $70 \mathrm{~km} \mathrm{~s}^{-1}$ between phases 0.062 and 0.558 . Such a decrease is also observed for $\ell$ Carinae (Fig. 4 of Nardetto et al. 2008). Thus, there is a relatively fast deceleration of the shock during its propagation in the upper atmosphere. The corresponding decrease of the shock energy is certainly the result of significant radiative losses of the shock.

\section{The transition phase}

For X Cygni, the main shock is first visible by means of the blueshifted absorption. It appears around phase 0.9. Then, a redshifted emission indicates the presence of the shock, which vanishes near phase 0.5 . Thus, the radiative shock is present in the atmosphere during half a pulsation period. At an average velocity of $85 \mathrm{~km} \mathrm{~s}^{-1}$, the shock front goes through about $82 \%$ of the stellar radius, if we take a photospheric radius of $105.2 R_{\odot}$ as suggested by Breitfellner \& Gillet (1993b). This means that the shock is well detached from the photosphere at phase 0.4 . When the P Cygni emission appears at phase 0.0, the shock front has already traveled $16 \%$ of the photospheric radius. This relatively small distance, however, appears sufficient to initiate the P Cygni profile.

During the short phase interval 0.9-0.0, there occurs the transition between the normal blueshifted emission (when the shock front is close to the photosphere) and the redshifted emission of the P Cygni profile. This transition is not clearly visible on X Cygni, because the blueshifted emission is never observed above the continuum. Convsersely, the transition is well observed on $\ell$ Carinae (see Fig. 4 of Nardetto et al. 2008). The beginning of the transition occurs abruptly. Indeed, at $\varphi=0.90$, the intensity of the blueshifted emission drops almost instantly under the continuum, while at the same time the depth of the redshifted absorption line increases greatly (Fig. 4 of Nardetto et al. 2008). These striking changes between the blue and red parts of the line profile, are perfectly synchronized. Consequently, the two types of emissions are not present at the same time. This means that they are not produced by two different shocks, but by the same shock. This also explains why only a single blueshifted absorption is visible when the P Cygni emission is present as shown by observations (Fig. 1). This is also consistent with the fact that a double peaked emission is never observed. Moreover, it is possible to understand why the velocity of the blueshifted absorption decreases with the pulsation phase, together with that of the emission of the P Cygni profile. Indeed, if we assume that energy losses of the shock due to the radiative cooling become the dominant process, then the shock velocity decreases during the rest of the outward propagation of the shock in the atmosphere.

\section{The $\mathrm{H} \alpha$ redshifted absorption line}

Near phase $\varphi=0.702$, a redshifted absorption appears within the $\mathrm{H} \alpha$ profile (Fig. 1). Its depth increases more and more. Its width is narrower than that of the photospheric absorption. It is clearly visible as an individual line until $\varphi=0.888$, after being blended with the photospheric absorption component centered at zero velocity. Its redshifted velocity slowly decreases from approximately $55 \mathrm{~km} \mathrm{~s}^{-1}(\varphi=0.702)$ to $47 \mathrm{~km} \mathrm{~s}^{-1} \quad(\varphi=0.062)$.

The redshifted absorption line appears long after the start of infalling movement, which occurs at $\varphi=0.40$ for metallic lines (Breitfellner \& Gillet 1993b). Already $60 \%$ of the infalling movement of layers, in which metallic lines are formed, is accomplished when the $\mathrm{H} \alpha$ absorption appears at $\varphi=0.702$. Of course, because hydrogen lines are formed higher in the atmosphere than metallic lines, $60 \%$ is certainly overestimated. It is striking that the redshifted absorption line appears only in the second and final part of the infall motion toward the photosphere. Indeed, the redshifted absorption is not observed until $\varphi=0.64$ (see the line profile at $\varphi=0.642$ ). It should be noticed that the pulsation phase $\varphi=0.64$ is the time of the minimum V-luminosity. Thus, until $\varphi=0.642$, we can conclude that the column density of the infalling gas is too small to contribute to the observation of an absorption line. This line will only be visible from $\varphi \gtrsim 0.70$, when the column density becomes sufficiently high. However, at this stage, when the absorption 
appears, its velocity around $55 \mathrm{~km} \mathrm{~s}^{-1}$ already has a supersonic value. This high velocity is the result of the gravitational acceleration of the gas since the beginning of the infalling motion at the maximum extension of the atmosphere $(\varphi=0.40)$. It is surprising that with a supersonic velocity, no emission is observed. Indeed, if an emission is present, it should appear as an inverse P Cygni profile, because the gas producing the redshifted emission is far from the photosphere. However, for a P Cygni profile, the emission is centered on the zero velocity, this means in practice that it is not easily detectable, because the emission is blended with the deep photospheric absorption also centered at zero velocity. Finally, in the phase interval $0.7-0.9$, when the $\mathrm{H} \alpha$ profile is observed as a nice double absorption, it should be the result of a blend between an inverse P Cygni profile and the photospheric absorption line.

Because the velocity of the redshifted absorption is observed to decrease from $55 \mathrm{~km} \mathrm{~s}^{-1}$ to $47 \mathrm{~km} \mathrm{~s}^{-1}$ between phases 0.702 and 0.062 , we can conclude that the motion of infalling layers is decelerating. This decrease in velocity $(\sim 15 \%)$ is certainly the consequence of the increasing density when layers approach the photosphere. Thus, the high atmosphere induces a strong compression on the photosphere. It is the reason why in long-period Cepheids, a bump is clearly observed in the light curve at the pulsation phase 0.8 (Breitfellner \& Gillet 1993b).

With an average velocity of $55 \mathrm{~km} \mathrm{~s}^{-1}$ during the appearance of the redshifted absorption $(\varphi=0.702)$ until the occurrence of the maximum of the bump in the light curve at $\varphi=0.79$, the infalling material travels about $40 \%$ of the thickness of the atmosphere between the maximum atmospheric extension and the maximum of the secondary acceleration. In the present work, the atmosphere is defined as the distance traversed by the main shock during its radiative phase (from $\varphi=0.9$ to $\varphi=0.4$ ). Consequently, the slowdown reaches a maximum intensity far above the photosphere, and if an emission is produced, it will necessarily form an inverse P Cygni profile.

\section{Origin of the two atmospheric accelerations}

For X Cygni, as shown by Breitfellner \& Gillet (1993b), there are two accelerations of the atmosphere by pulsation cycle. The primary acceleration has its maximum when the radius reaches its minimum value. We observe the same behaviour in the shortperiod Cepheid $\delta$ Cephei (Breitfellner \& Gillet 1993a), except that the primary acceleration occurs earlier $(\Delta \varphi=0.06)$. For $\mathrm{X}$ Cygni, the maximum of the primary acceleration is about two times larger than the mean stellar gravity, while it is of the same order for $\delta$ Cephei or other short-period Cepheids.

A secondary acceleration is observed for X Cygni near $\varphi=$ 0.79 (Breitfellner \& Gillet 1993b). Its intensity is large because it reaches $75 \%$ that of the primary acceleration. $\delta$ Cephei does not show a secondary acceleration (Breitfellner \& Gillet 1993a). The maximum of the secondary bump of the light curve occurs also at $\varphi=0.79$. Consequently, these two exactly synchronized features are probably the consequence of the strong atmospheric compression provoked by the infalling motion of the high atmosphere on the photosphere. As shown by the radius variation curve (Breitfellner \& Gillet 1993b), the infalling motion is notably slowed down. This phenomenon does not induce an appreciable change within the $\mathrm{H} \alpha$ profile near $\varphi=0.79$.

Actually, the slowdown begins just after the occurrence of the minimum luminosity. It increases gradually until the bump maximum at $\varphi=0.79$. Therefore, the slowdown is not a sudden and violent process caused by the collision between the fastest upper layers of the atmosphere and the photosphere. This slowdown induces a local compression above the photosphere. It strongly decreases the infalling motion up to a constant velocity near $\varphi=0.85$. The layers finally stop moving and then sharply reverse their movement when the main shock emerges from the photosphere and quickly crosses the atmosphere (main acceleration).

\section{Episodes of turbulence amplification}

The observed line widths of metallic lines cannot be explained by the effects of pulsation, temperature variation, and rotation alone. It is assumed that gas turbulence is a necessary mechanism to be taken into account to explain observations. It is even the dominant broadening mechanism when enlargement of the pulsation amplitude is high.

The turbulent velocity (microturbulent velocity) is determined as the difference between the observed line width and that of a calculated line profile, using a nonlinear nonadiabatic radial pulsation model, which is more or less sophisticated (e.g. Breitfellner \& Gillet 1993a; Fokin et al. 1996). In general, the rotational velocity of the star, which is a necessary parameter used to estimate the theoretical line profile, is unknown. More generally, there is a great uncertainty on $V_{\text {rot }} \sin i$ and only a rough estimate can be given.

As observed, the turbulence is minimal when the star reaches its maximum radius, because the atmosphere is in its best quiet hydrodynamic state. Then, during the free infalling motion, the atmospheric gas volume decreases and consequently, the temperature increases. Thus, when the atmosphere begins to contract from its maximum extension, the turbulent velocity increases (Fig. 4 of Breitfellner \& Gillet 1993b). Owing to the reaction of the atmospheric layers to the gravitational compression, from a maximum value of $-38.3 \mathrm{~km} \mathrm{~s}^{-1}$ at $\varphi=0.73$, the infalling velocity begins to decrease more quickly. This corresponds to the appearance of the secondary acceleration and the turbulent velocity increases more rapidly. Always caused by the reaction of the atmosphere to the compression, the acceleration reaches a maximum, before decreasing and vanishing. At that time $(\varphi=0.85)$, the infalling velocity is small $\left(-12 \mathrm{~km} \mathrm{~s}^{-1}\right)$ and almost constant. Thus, the compression rate decreases, as does the turbulent velocity. Finally, during the maximum of the secondary acceleration, a peak of compression is produced above the photosphere. It is the result of the infalling motion of the highest and fastest layers on the deepest and slowest ones. It is in this sense that we can speak of a collision, but in fact, it is instead a sudden compression of the gas well above the photosphere.

It is always at that time $(\varphi=0.85)$, that the main shock is traversing the photosphere, causing the primary acceleration and the sudden reversal of the movement of all atmospheric layers. The velocity turbulent curve (Fig. 4 of Breitfellner \& Gillet 1993b) clearly shows that the main shock yet again increases the turbulence velocity, but not as effectively as during the secondary acceleration. As shown by Fokin et al. (1996) for $\delta$ Cephei, at $\varphi=0.85-0.95$ the main shock does not reach a high Mach number $(M \lesssim 4)$. Consequently, the compression rate induced by the shock is certainly modest and may explain why the rate of turbulence is smaller than that achieved during the secondary acceleration. Moreover, as shown by numerical simulations (Rotman 1991), the amplification rate decreases with increasing initial turbulent energy. Thus, the smaller turbulent velocity observed during the primary acceleration may also, in part, be the result of the first turbulence amplification provoked by the atmospheric compression during the secondary acceleration. 
Breitfellner \& Gillet (1993b) explained the first peak of the turbulent velocity by a shock compression much larger than that occurring in the classical shock adiabatic case. Indeed, the shock being radiative, it induces a compression rate larger than in the classical limit case (4 for an atomic gas). This explanation implies that the turbulence increase must be rapid and not gradual, as observed. However, as already mentioned, the intensity of the shock does not seem high at this pulsation phase and consequently, a large shock compression is not expected. It also seems difficult that the shock itself can induce the large bump observed on the V-light curve because, in this photometric band, the shock luminosity is probably smaller than that produced by the photosphere (Gillet 2013).

\section{Comparison with other radially pulsating stars}

\subsection{Classical Cepheid stars}

Nardetto et al. (2008) report very high-resolution spectroscopy $(R=120000)$ of eight galactic Cepheids with a good period sampling $(3.39 \mathrm{~d} \leq P \leq 41.52 \mathrm{~d})$. The star $\ell$ Carinae was observed as already discussed above. A $2 \mathrm{D}$ (wavelength versus pulsation phase) map of the $\mathrm{H} \alpha$ profile of three short-period Cepheids - R TrA (3.4 d), S Cru (4.7 d), and Y Sgr (5.8 d) - show very similar profile varaiations from one star to the next and the $\mathrm{H} \alpha$ line profile behaves similarly to metallic absorption lines. No emission components (blueshifted or redshifted) above the continuum are observed. Nevertheless, a blueshifted absorption component, like the one seen in X Cygni, is observed. It disappears suddenly near $\varphi=0.75-0.80$, while the deep photospheric absorption, after reaching its largest redshifted velocity, suddenly shifts on the blue side of the profile. This sudden jump in the evolution of the central absorption of the $\mathrm{H} \alpha$ profile corresponds to the transition episode between the end of the infalling motion of the atmosphere and the rapid passage of the main shock throughout the photosphere. For short-period Cepheids, there is no spectral indication in $\mathrm{H} \alpha$ that the shock is detached from the photosphere (appearance of a P Cygni profile).

The case of medium-period Cepheids (around 10 days) is particular, because these stars are very close to the $P_{2} / P_{0}=0.5$ resonance between the fundamental and the second overtone modes. Thus, we observe the transition phase discussed above, in addition to the disturbance event due to the $P_{2} / P_{0}=0.5$ resonance near $\varphi=0.50$.

Nardetto et al. (2008) report time series of $\mathrm{H} \alpha$ line profiles for the long-period Cepheids - RZ Vel (20.4d), $\ell$ Car (35.6d), and RS Pup (41.5 d). As for X Cygni $(P=16.4 \mathrm{~d})$, the evolution of these profiles are similar to each other except for the presence or absence of blueshifted and redshifted emission components. The presence of an emission means that the intensity of the main shock wave is particularly strong. In long-period Cepheids, the intensity of the main shock seems higher than in short-period Cepheids because $\mathrm{H} \alpha$ is never observed in emission in the shortperiod of stars. This is also consistent with period-amplitude diagrams. Indeed, the amplitude of long-period Cepheids up to approximately $17 \mathrm{~d}$ increases dramatically, both in luminosity and radial velocity, when the period increases (Klagyivik \& Szabados 2009). However, when $P>17 \mathrm{~d}$, because the convective transport becomes effective at a certain depth in subphotospheric layers, the pulsation motion is gradually damped, and consequently the pulsation amplitude decreases. Thus, in very long-period Cepheids $(P>17 \mathrm{~d})$, the main shock must reach its full development only in the upper layers of the atmosphere i.e. in extremely low gas density regions and not in dense layers as in short-period Cepheids. This is also consistent with the fact that we observe $\mathrm{H} \alpha$ P Cygni profiles both in $\mathrm{X}$ Cygni $(P=16.4 \mathrm{~d})$ and $\ell$ Carinae $(P=35.6 \mathrm{~d})$. However, we expect that the main shock must be stronger for $\ell$ Carinae than for X Cygni because a blueshifted emission above the continuum occurs only in $\ell$ Carinae.

\subsection{RR Lyrae stars}

The detailed $\mathrm{H} \alpha$ profile variations of RR Lyrae stars were for example given by Gillet \& Crowe (1988). Recently, Gillet \& Fokin (2014) have shown that the evolution of the different emission components observed within the $\mathrm{H} \alpha$ profile of RR Lyrae is the natural consequence of the large extension of the expanding atmosphere. When the shock is close to the photosphere, then the receding part of the shock producing the redshifted emission component is occulted by the stellar disk. In this case, the shock emission is observed as a blueshifted component within the large photospheric absorption. Then, when the shock propagates in the atmosphere, its receding part becomes increasingly visible and the emission component shifts to the red side of the profile, which gives a P Cygni profile. In fact, we find the same scenario occurs in long-period Cepheids. Finally, these changes in profile types, from blueshifted to redshifted emission, result naturally from the propagation of a strong shock wave in a radially pulsating atmosphere.

\subsection{W Virginis stars}

The detailed $\mathrm{H} \alpha$ profile variations of $\mathrm{W}$ Virginis were, for example, given by Lèbre \& Gillet (1992). From his hydrodynamical pulsation model of $\mathrm{W}$ Virginis, Fokin finds that W Viginis has a very extended and dense atmosphere (Kovtyukh et al. 2011). Thus, unlike X Cygni and $\ell$ Carinae or RR Lyrae stars, the envelope of W Virginis should be optically thick in the $\mathrm{H} \alpha$ line core. In this case, as shown by Bertout \& Magnan (1987), the line profile from fast-moving spherical shells, such as those induced by shock waves travelling through stellar envelopes, is not a $\mathrm{P}$ Cygni but a parabolic profile. It is the reason why a $\mathrm{P}$ Cygni profile is never observed in W Virginis stars, although this line is in emission throughout the pulsation cycle (Lèbre \& Gillet 1992). However, the envelope should be optically thin for metallic absorption lines, because inverse P Cygni profiles of Ba II, Ti I, Fe I, Fe II, etc., were observed from $\varphi=0.67$ to 0.06 (Kovtyukh et al. 2011).

\section{Conclusion}

For several decades, high-quality spectral observations of longperiod Cepheids, especially $\mathrm{H} \alpha$ profile variations, have indicated that the dynamics of the atmosphere of these stars is extremely difficult to understand. Recent pulsation models show the presence of strong shock waves and large velocity gradients in the atmosphere. Using both observationnal and theoretical results, we have provided for the first time a full picture of the dynamical structure of the atmosphere of long-period Cepheids.

First, when the shock is emerging from the photosphere, its receding part is occulted by the stellar disk and consequently, only the advancing part of the shock can be observed. If an emission is produced in the shock wake, then it is blueshifted. Later, during the shock propagation in the atmosphere, if the emission persists, then it is redshifted. More precisely, because the shock is then detached from the photosphere, a P Cygni profile is 
formed. This implies that the gas is not yet completely optically thick. The relatively rapid transition between these two spectroscopic situations is clearly observed on the $\mathrm{H} \alpha$ profile near the luminosity maximum. When the shock intensity is not high enough to induce a blueshifted emission, an absorption component is observed, as in X Cygni.

Finally, our explanation of the occurrence of P Cygni emission profile in $\mathrm{H} \alpha$ is a general process in atmospheric dynamics, when the opacity of the gas is moderate. When the atmospheric gas becomes optically thick, P Cygni profiles are no longer observed throughout the propagation of the shock in the atmosphere. Moreover, the presence of a redshifted absorption, which appears after the minimum brightness and disappears after the maximum brightness, is observed in the three types of pulsating stars discussed above.

Acknowledgements. I want to thank Andrei Fokin for sending us additional information about his pulsating model of $\ell$ Carinae. I also wish to acknowledge Philippe Mathias for pertinent comments and suggestions. I gratefully acknowledge Helenka Kinnan for her very careful reading of the paper.

\section{References}

Abt, H. A. 1978, PASP, 90, 309

Abt, H. A. 1959, ApJ, 130, 824

Baker, N., \& Kippenhahn, R. 1965, ApJ, 142, 868

Baldry, I. K., Taylor, M. M., Bedding, T. R., et al. 1997, MNRAS, 289, 979

Bertout, C., \& Magnan, C. 1987, A\&A, 183, 319

Breitfellner, M. G. 1993, Ph.D. Thesis, University of Vienna

Breitfellner, M. G., \& Gillet, D. 1993a, A\&A, 277, 524

Breitfellner, M. G., \& Gillet, D. 1993b, A\&A, 277, 553

Butler, R. P., \& Bell, R. A. 1997, ApJ, 480, 767

Caldwell, J. A. R., \& Coulson, I. M. 1987, AJ, 93, 1090

Castor, J. P. 1967, Ph.D. Thesis, California Institute of Technology, Pasadena, California

Castor, J. P. 1972, The Evolution of Population II Stars, Proc. Conf. held at the State University of New York at Stony Brook, December, 1970, ed. A. G. D. Philip, Dudley Observatory Report No. 4, 147

Christy, R. F. 1964, Rev. Mod. Phys., 36, 555
Christy, R. F. 1966, ApJ, 144, 108

Christy, R. F. 1967, IAU Symp., 28, 105

Fadeyev, Yu. A., \& Gillet, D. 2004, A\&A, 420, 423

Fokin, A. B. 1991, MNRAS, 250, 258

Fokin, A. B. 1992, MNRAS, 256, 26

Fokin, A. B., \& Gillet, D. 1994, A\&A, 290, 875

Fokin, A. B., \& Gillet, D. 1997, A\&A, 325, 1013

Fokin, A. B., Gillet, D., \& Breitfellner, M. G. 1996, A\&A, 307, 503

Fokin, A. B., Nardetto, N., \& Mathias, P. 2009, EAS Pub. Ser., 38, 151

For, B.-Q., Preston, G. W., \& Sneden, C. 2011, ApJ, 194, 38

Geroux, C. M., \& Deupree, R. G. 2011, ApJ, 731, 18

Geroux, C. M., \& Deupree, R. G. 2013, ApJ, 771, 113

Gillet, D. 2013, A\&A, 554, A46

Gillet, D., \& Crowe, R. A. 1988, A\&A, 199, 242

Gillet, D., \& Fokin, A. B. 2014, A\&A, 565, A73

Gillet, D., Fokin, A. B., Breitfellner, M. G., et al. 1999, A\&A, 344, 935

Gillet, D., Fabas, N., \& Lèbre, A. 2013, A\&A, 553, A59

Hill, S. J. 1972, ApJ, 178, 793

Hutchinson, J. L., Hill, S. J., \& Lille, C. F. 1975, AJ, 80, 1044

Kervella, P., Mérand, A., \& Gallenne, A. 2009, A\&A, 498, 425

Kiss, L. L., \& Vinkó, J. 2009, MNRAS, 314, 420

Klagyivik, P., \& Szabados, L. 2009, A\&A, 504, 959

Kovtyukh, V. V., Wallerstein, G., Andrievsky, S. M., et al. 2011, A\&A, 526, A116

Krap, A. H. 1960, in Stellar Atmospheres, ed. J. L. Greenstein (Chicago: University of Chicago Press), 370

Lèbre, A., \& Gillet, D. 1992, A\&A, 255, 221

Moffett, T. J., \& Barnes III, T. G. 1987, PASP, 99, 1206

Nardetto, N., Groh, J. H., Kraus, S., et al. 2008, A\&A, 489, 1263

Neilson, H. R., \& Lester, J. B. 2008, ApJ, 684, 569

Preston, G. W. 1964, ARA\&A, 2, 23

Preston, G. W. 2009, A\&A, 507, 1621

Preston, G. W. 2011, AJ, 141, 6

Preston, G. W., \& Paczynski, B. 1964, ApJ, 140, 181

Preston, G. W., Smak, J. \& Paczynski, B. 1965, ApJS, 12, 99

Rotman D., 1991, Phys. Fluids A3, 1792

Sanford, R. 1949, A\&A, 109, 208

Schwarzschild, M. 1952, Transaction of the IAU VIII, ed. P. T. Oosterhoff (Cambridge University Press), 811

Tuggle, R. S., \& Iben, I. J. 1973, ApJ, 186, 593

Wallerstein, G. 1959, ApJ, 130, 560

Wallerstein, G. 1983, PASP, 95, 422

Wilson, R. E. 1953, in General Catalogue of Stellar Radial Velocities (Carnegie Inst. Washington D.C. Publ.), 601 